\title{
Modelling and Control of Surge in Centrifugal Compression Based on Fuzzy Rule System
}

\author{
Ahmed Hafaifa, Attia Daoudi, Kouider Laroussi \\ Industrial Automation and Diagnosis Systems Laboratory, \\ Department of Science and Technology, \\ Science and Technology Faculty, \\ University of Djelfa 17000 DZ Algeria. \\ hafaifa@hotmail.com; a_daoudi@hotmail.com; kouider-laro@hotmail.com
}

\begin{abstract}
This paper presents the problem of robust design of surge controller in centrifugal compression system using fuzzy techniques. The use of fuzzy techniques in modelling and control has been important to reduce costs and losses of the system. In this paper, based on the fuzzy modelling and control, a new way to establish fuzzy logic controller is proposed. In this paper, the performance of the fuzzy controller has been designed to give a new control technique of surge in centrifugal compression system. The validation results show the effectiveness of the proposed approach in order to achieve desired performance.
\end{abstract}

Keywords: Compression system, centrifugal compressor, surge control, supervision system, fuzzy modelling, fuzzy control, surge phenomena.

\section{Introduction}

In recent years fuzzy logic has been an active area of research, many research efforts have been focused on fuzzy modelling and control issues based on the Takagi-Sugeno (TS) fuzzy model [2][3][8] and [12], which is described by fuzzy IF-THEN rules. For a nonlinear system that is transformed successfully into a T-S fuzzy model, the stability of the overall nonlinear system still cannot be guaranteed even if each subsystem of the T-S fuzzy model is stable. In this work, we propose a new fuzzy controller design for the supervision of a dynamical complex system, based on dynamic fuzzy model for control and supervision. The idea of model based fuzzy observers in supervision is to compare output signals of the model with the real measurements available in the process, thereby generating the indicators, which are fault indicators giving information about the location and timing of a fault.

This fuzzy supervisory approach requires precise mathematical relationships relating the model to the process, to allow the detection of small abrupt and incipient faults quickly and reliably. Many standard observer-based techniques exist in the literature providing different solutions to both the theoretical and practical aspects of supervision problem for linear and nonlinear system [1], [6] and [13]. Many of these procedures are based on the design of an unknown input observer robust with respect to the disturbances. If the disturbances and modelling errors are not properly taken into account in the estimation process, it is then likely that any attempt in monitoring the system's health based on the observer leads to numerous false alarms. From this issue, many results had been obtained in order to get a representation of non linear systems under the form of multiple linear sub-models aggregated by fuzzy logic [9].

This paper describes tools and techniques that can and have been used to model transient flows and performance, mechanical and the minimum response time in compressor system control. The tools used by fuzzy logic include a method of characteristic transient flow analysis routine and finite time step programs that simulate control systems, valve actuators, and the opening (or closing) rate of valves with the resulting flows. The effects of volumes and lengths of station piping, scrubbers, and coolers including temperature effects are accounted for. Fuzzy logic models and control also track the performance of centrifugal compressors at different speeds, account for the rotation inertia of compressor trains, and evaluate the thermo physical properties of gas streams.

\section{Gas Compression System}

In this work, we develop the strategy of fuzzy modelling and control in the area of surge supervision in centrifugal compression gas system. For this reason, the hardware and 
software are considerably different than what would be used in a complex application, where cost would be a driving factor and economies of scale would allow for custom hardware and optimized software. The plant is a real compression system, the station of the gas compression SC3 of SONATRACH located in Algeria. It consists on a centrifugal compressor, close coupled valve, compressor duct, plenum volume and a throttle. The throttle can be regarded as a simplified model of a turbine. The system is showed in Figures 1.a, 1.b, 1.c, 1.d and 1.e.

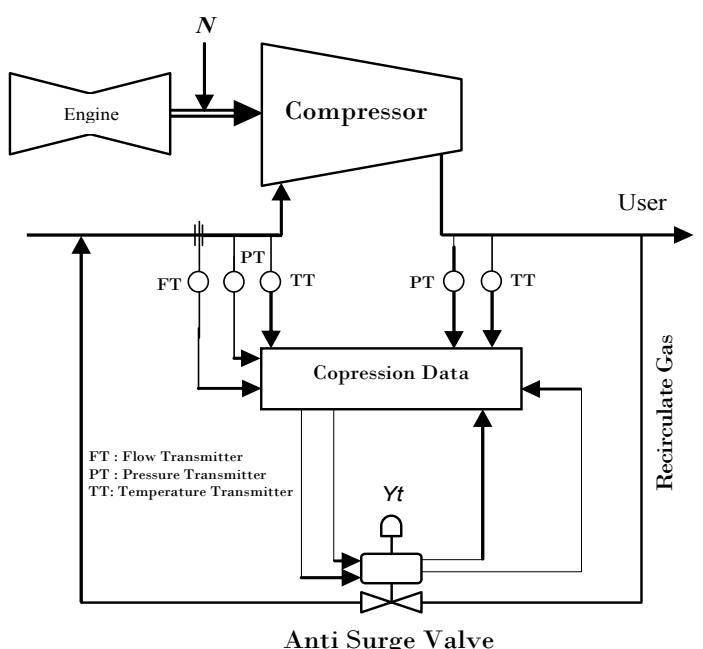

Figure 1.a. Compression system

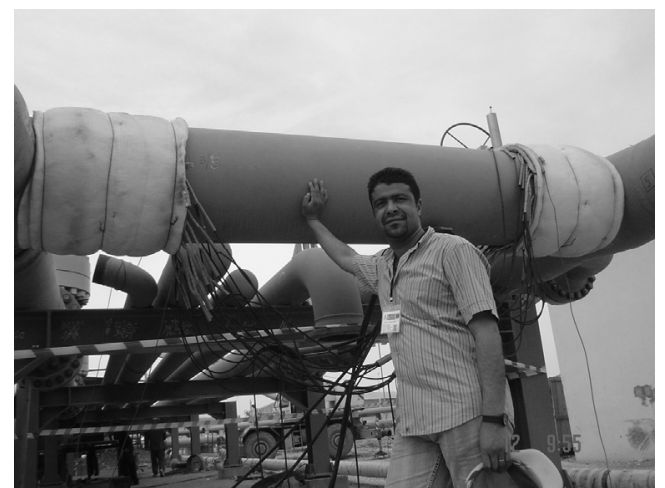

Figure 1.b. Gas pipeline in compression system

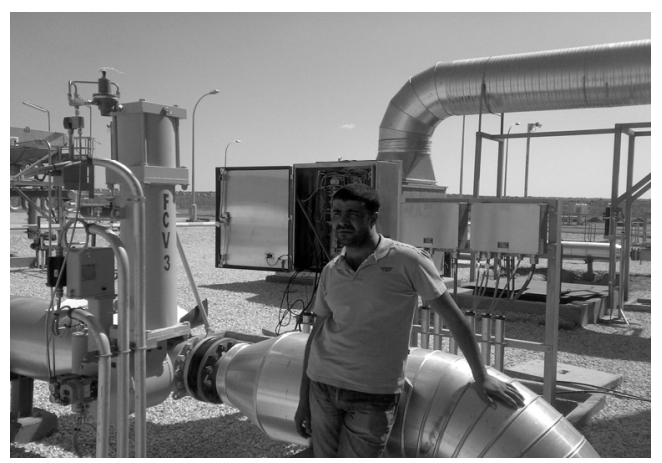

Figure 1.c. Anti-surge valve used in compression system

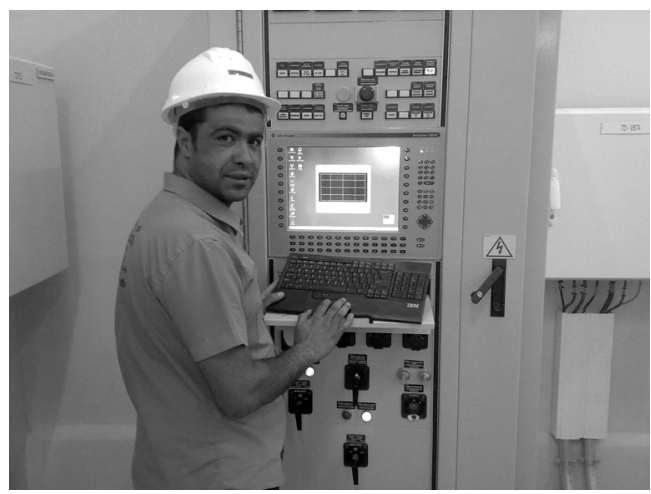

Figure 1.d. Compression station data in compression system

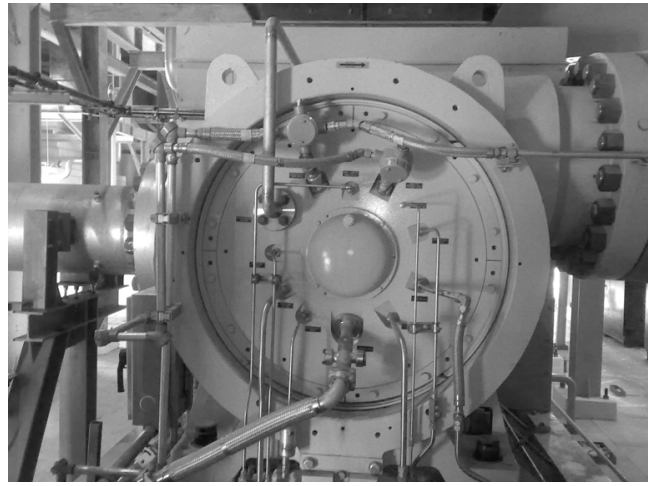

Figure 1.e. Centrifugal compressor in compression system

In this work, we consider a special class of nonlinear systems for fuzzy modelling and control purposes. The use of fuzzy logic makes it possible to devise the supervision scheme based on the incidence matrix. To illustrate the efficiency of the proposed fuzzy method, experimental results of the fuzzy supervision for a compression system are provided. We note that this work is a first step in accessing the factors that affect the success of surge control in natural gas pipeline compression system [8], [9], [10], [11], [12], [13] and [14].

Centrifugal compressors will surge when forward flow through the compressor can no longer be maintained, due to an increase in pressure across the compressor, and a momentary flow reversal occurs. Once surge occurs, the reversal of flow reduces the discharge pressure or increases the suction pressure, thus allowing forward flow to resume again until the pressure rise again reaches the surge point.

Surge is characterized by large amplitude fluctuations of the pressure and by unsteady, but circumferentially uniform, annulusaveraged mass flow. This essentially one 
dimensional instability affects the compression system as a whole and results in a limit cycle oscillation in the compressor map.

This surge cycle will continue until some change is made in the process or compressor conditions. Figure 2 shows a pressure trace for a compressor system, which was initially operated in a steady operating point. By throttling the compressor mass flow, the machine is run into surge. This figure illustrates the difference between pressure variations before and after surge initiation. A surge controller typically measures a function of pressure rise versus flow. The controller operates a surge valve to maintain sufficient forward flow to prevent surge [5], [6] and [15].

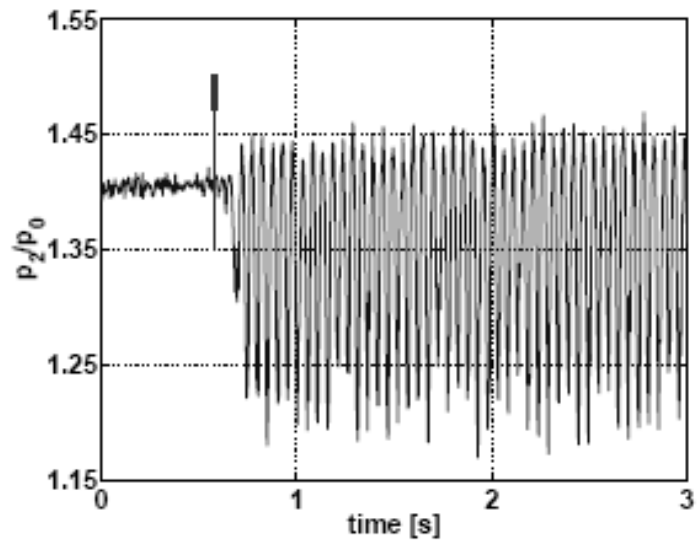

Figure 2. Surge initiation in compression system

In this paper we use the Greitzer model proposed in [5], [6], [7], [17], [18] and [23] to describe the dynamic behaviour of the compression system. This model is given by:

$$
\left\{\begin{array}{l}
\dot{p}_{p}=\frac{a_{01}^{2}}{V_{P}}\left(m-m_{t}\right) \\
\dot{m}=\frac{A_{1}}{L_{C}}\left(p_{2}-p_{p}\right) \\
\dot{N}=\frac{1}{2 J \pi}\left(\tau_{t}-\tau_{C}\right)
\end{array}\right.
$$

where $m$ is the compressor mass flow, $m_{t}$ is the throttle flow, $p_{p}$ is the plenum pressure, $p_{2}$ is the pressure downstream of the compressor, $a_{01}$ is the inlet stagnation sonic velocity, $V_{p}$ is the plenum volume, $A_{1}$ is the area of the impeller eye (used as reference area), $L_{c}$ is the length of compressor and duct, $N$ is the spool moment of inertia, $J$ is the inertia of all rotating parts, $\tau_{t}$ is the drive torque and $\tau_{c}$ is the compressor torque.

\section{Fuzzy Modelling}

Nowadays practical solutions of engineering problems involve model-integrated computing. This integration means that the available knowledge is represented in a proper form and acts as an active component of the procedure (computer program) to be executed during the operation of the diagnostics, measuring, control, etc. devices. Thus, model based approaches offer a very challenging way to integrate a priori knowledge into the procedure. Fuzzy logic provides a formal framework for constructing systems exhibiting both good numeric performance (precision) and linguistic representation (interpretability). From a numeric point of view, fuzzy systems exhibit nonlinear behavior and can handle imprecise and incomplete information. Linguistically, they represent knowledge in the form of rules, a natural way for explaining decision processes [2], [4].

Fuzzy modelling is an arduous task, demanding the identification of many parameters. This paper analyses the fuzzymodelling problem and different approaches to coping with it, focusing on evolutionary fuzzy modelling, and the design of fuzzy inference systems using evolutionary algorithms and different fuzzy operations (shown in the Figure 3), which constitute the methodological base of our approach. In order to promote this analysis the parameters
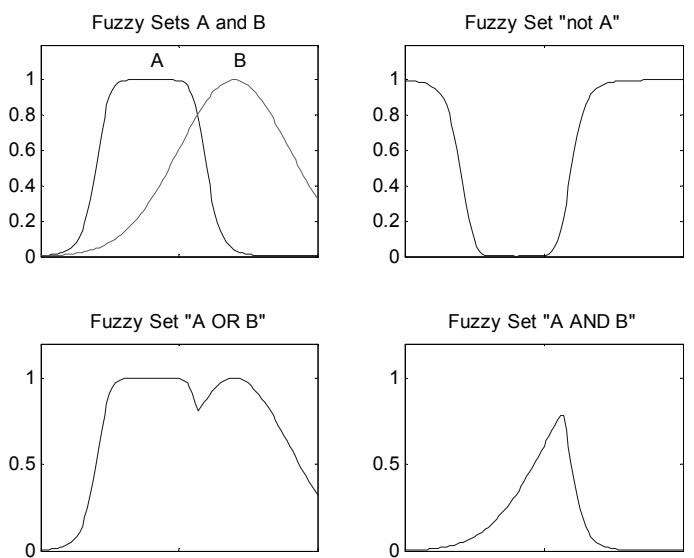

Figure 3. Different fuzzy operations

of a fuzzy system are classified into four categories: logic, structural, connective, and operational. In this paper an intelligent and flexible fuzzy modelling approach is introduced which is suitable for the 
approximate modelling of complex systems opening a way for the fault diagnosis and isolation in the system. The different variables of output system are defined by one of the membership functions shown in the Figure 4. area of application and the conflict existing in the overlapped areas [1], [3]. In this way, TS fuzzy systems present the following interesting features: locality, smooth switching and existence of mathematical tools for system design.

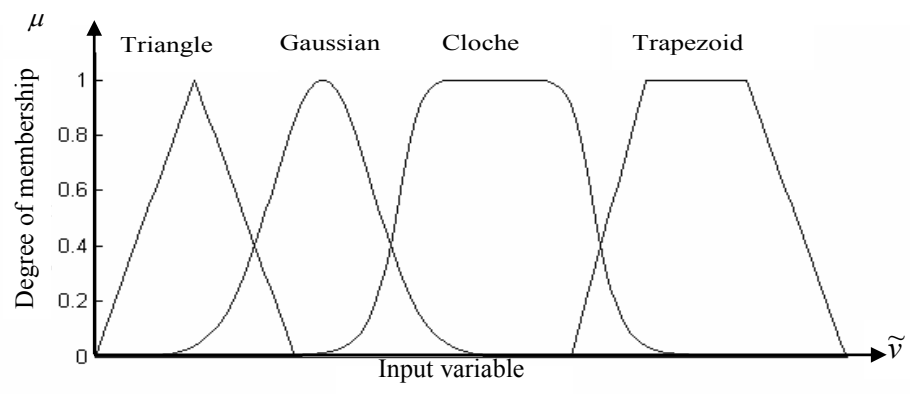

Figure 4. Membership functions

\subsection{Takagi-Sugeno fuzzy model}

A dynamical complex and nonlinear system can be approximated by a Takagi-Sugeno (TS) fuzzy model. In [2], Takagi, Sugeno presented a mathematical tool to procure a fuzzy model of a system. The TS fuzzy model consists of a set of IF-THEN rules which represent the local linear input and output relations of a nonlinear system based on rules usually presenting the following structure:

$$
\begin{aligned}
& R_{i} \text { : If } \mathrm{X}_{1} \text { is } \mathrm{A}_{\mathrm{i} 1} \text { and } \ldots \mathrm{X}_{\mathrm{n}} \text { is } \mathrm{A}_{\mathrm{in}} \text { Then } \\
& \qquad \mathrm{Y}=p_{i 1} \cdot \mathrm{X}_{1}+\ldots+\mathrm{p}_{\mathrm{in}} \cdot \mathrm{X}_{\mathrm{n}}+p_{i o}
\end{aligned}
$$

where $X_{i}$ are the system input variables and $Y$ is the system output variable that determines a local linear input-output relation by means of the real-valued coefficients $p_{i j}$. The output of a fuzzy relation based system considering a knowledge base composed of $m$ TS rules is computed as the weighted average of the individual rule outputs given by:

$$
Y_{i}=\frac{\sum_{i=1}^{m} h_{i} y_{i}}{\sum_{i=1}^{m} h_{i}}
$$

With $h_{i}=T\left(A_{1}\left(X_{1}\right), \ldots, A_{n}\left(X_{n}\right)\right)$ being the matching degree between the antecedent part of the $i$-th rule and the current system inputs $\left(X_{1}, \ldots, X_{\mathrm{n}}\right)$, and with $T$ being a t-norm of fuzzy logics, in such a way that references to real numbers are eliminated from definitions and theorems, being removed to the standard semantics of fuzzy logic. As seen, these partial relations are combined by aggregation, taking into account their dominance in their respective
The main feature of TS fuzzy systems is to express the local property of each rule by a linear function. So, a continuous time TS model is described by a set of If...Then rules, where the $i$-th rule takes the form [20], [21] and [22]:

Rule i:

If $x_{1}$ is $M_{i 1}$ and $x_{2}$ is $M_{i 2}$ and . . and $x_{n}$ is $M_{\text {in }}$

Then: $\sigma(x)=A_{i} x+b_{i}$

Where: $x=\left[\begin{array}{llll}x_{1} & x_{2} & \ldots & x_{\mathrm{n}}\end{array}\right]$ is the state vector, $i=1, \ldots, \mathrm{r}, r$ is the number of rules, $\sigma$ are the local output variables, $b_{i}$ are the real-valued parameters, $A_{i}$ is an $n \times n$ matrix and $M_{i j}$ are the fuzzy sets for inputs. Using weighted average defuzzifiers, the aggregated fuzzy model is given by:

$\sigma(x)=\frac{\sum_{i=1}^{r} w_{i}(x)\left(A_{i} x+b_{i}\right)}{\sum_{i=1}^{r} w_{i}(x)}$

Where $w_{i}$ is defined as:

$w_{i}(x)=\prod_{i=1}^{n} \mu_{i j}\left(x_{j}\right)$

And $\mu_{i j}$ is the membership function of $j-t h$ fuzzy set in the $i-t h$ rule. Now, defining:

$$
\alpha_{i}(x)=\frac{w_{i}(x)}{\sum_{j=1}^{r} w_{j}(x)}
$$

We can write: 
$\sigma(x)=\sum_{i=1}^{r} \alpha_{i}(x)\left(A_{i} x+b_{i}\right)$

with: $\alpha_{i}$ is such that $\alpha_{i} \geq 0$ and $\sum_{i=1}^{r} \alpha_{i}=1$

(a) Zadeh's Arithmetic Rule
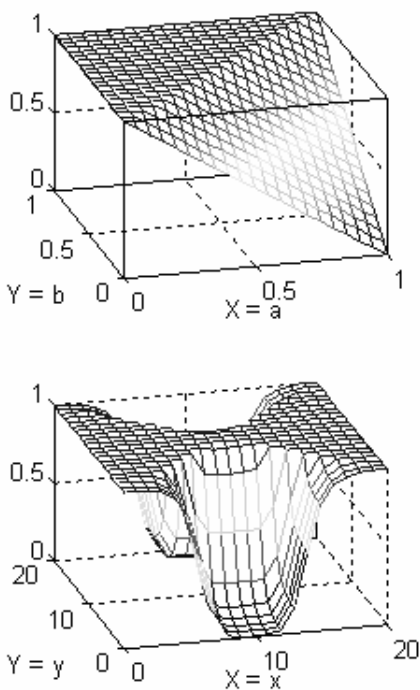

- Inference engine: which, by simulating a human decision process, infers the fuzzy control action from the knowledge of the control rules and the linguistic variable definitions; (b) Zadeh's Max-Min Rule
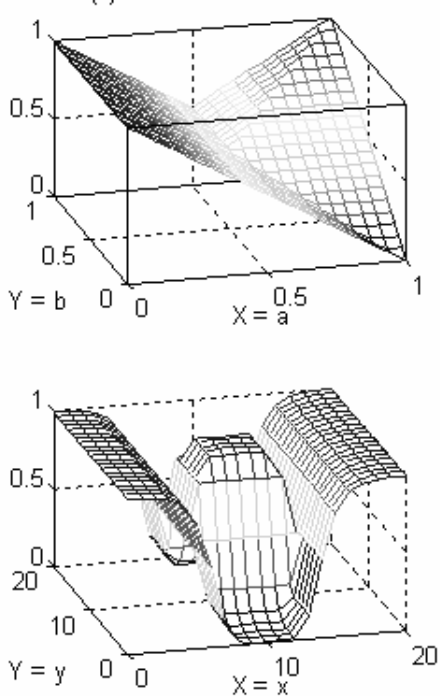

Figure 5. Fuzzy implications used in TS fuzzy model

The system defined by the equation (8) is generally nonlinear. The interpretation of equation (8) is that the overall system is a fuzzy blending of the given implications. In this work, Zadeh max-min rule and arithmetic rule implications, shown in Figure 5, enable us to deduce indicators from TS fuzzy model.

\section{Fuzzy Controller}

A Fuzzy logic controller can be handled as a system that transmits information like a conventional controller with inputs containing information about the plant to be controlled and an output that is the manipulated variable. The Control systems with fuzzy controllers are often successfully applied in practice. Their great advantage is the possibility to introduce the knowledge of human experts about proper and correct control of a plant in the controller [16], [19]. The general structure of a fuzzy logic control is represented in Figure 6 and comprises four principal components:

- fuzzyfication interface: which converts input data into suitable linguistic values;

- Rule base: which consists of a data base with the necessary linguistic definitions and the control rule set;
- defuzzyfication interface: which yields a nonfuzzy control action from an inferred fuzzy control action.

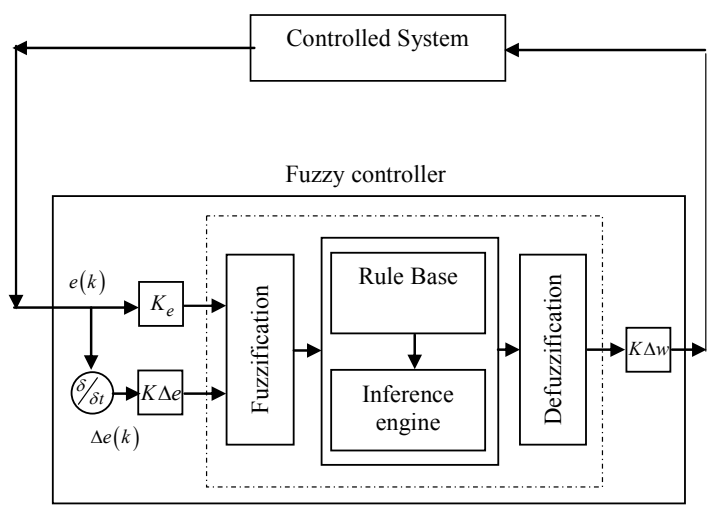

Figure 6. Basic structure of a fuzzy controller

The classical controller development approach relies on a linearization of the system dynamics and on the application of a standard controller (PID, PI) to compensate the effects of the non modelled non linearities. By this approach certain system is stabilised close to its nominal operating point. Problems could appear when the set point is changeable within wide operating range, as it is the case for our application in compression system. Fuzzy controllers have become popular in recent years because they do not necessarily require a theoretical model of the 
plant which is to be controlled. Therefore, in order to develop a fuzzy controller, one needs to first have access to a human expert, find quantifiable means to present the expert's experience, and determine a mapping from itself consists of three stages: fuzzification, fuzzy inference machine and defuzzification. A schematic representation of the proposed surge control system using fuzzy controller is given in figure 7.

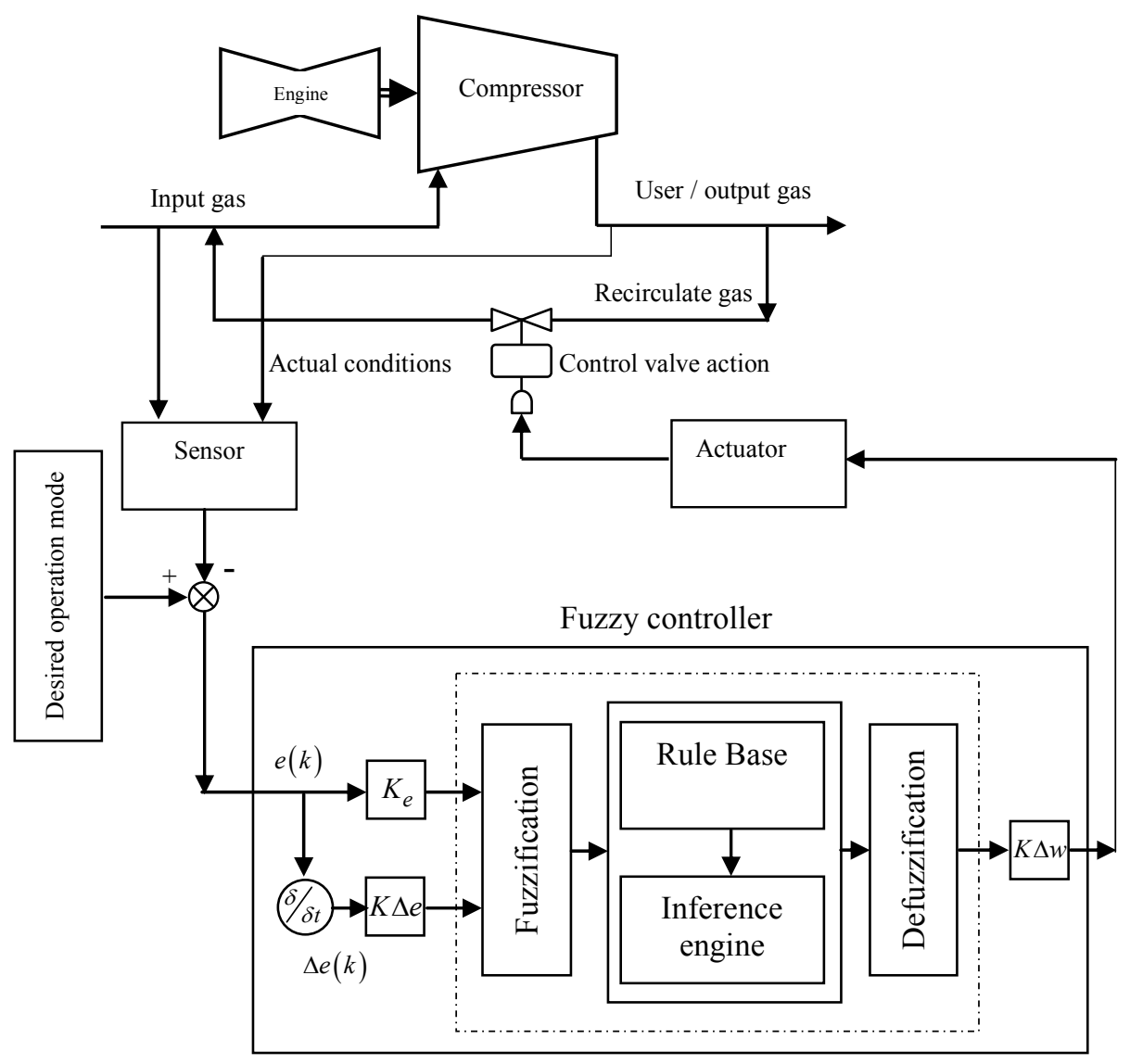

Figure 7. Schema of fuzzy controller used for surge control system

states of the plant to the fuzzy measures with which the expert's knowledge is quantified.

In this paper, fuzzy logic is used to surge control in compression system. To manipulate and measure system behavior, the fuzzy control of compression system should include sensors that provide information about the momentary flow conditions and actuators to influence those conditions, which determine the appropriate control action after comparing measured data with the desired flow conditions. The fuzzy controller proposed in this work has three inputs: the pressure downstream of the compressor $p_{p}$ normalized by the pressure coefficient $\Psi$, the compressor mass flow $m$ normalized by the mass flow coefficient $\Phi$, the spool moment of inertia $N$ and one output :desired actuator force $w$ applied to the anti surge valve given by the throttle opening $\gamma_{V}$. The control system

\section{Application Results}

The objective of this part of experimental results is to investigate the fuzzy controller described in this work to the protection of the centrifugal compression gas system. The surge stabilization using the proposed fuzzy techniques is experimentally investigated in the examined compression system (gas compression station in Algeria $\mathrm{SC} 3 /$ Sonatrach). This station has control room and computer equipment, which allowed us to make measurements of inputs and outputs of our compression system, to allow the application of the control algorithm based on analytical models and especially fuzzy supervisory system in real time from a station data implemented on site.

The figure 8 shows the response of the compression system for the mass flow input 
and the associate residual, there is a value that the residuals consistently exceed the threshold of detection, this figure show a case where a surge is detected in the examined compression system.
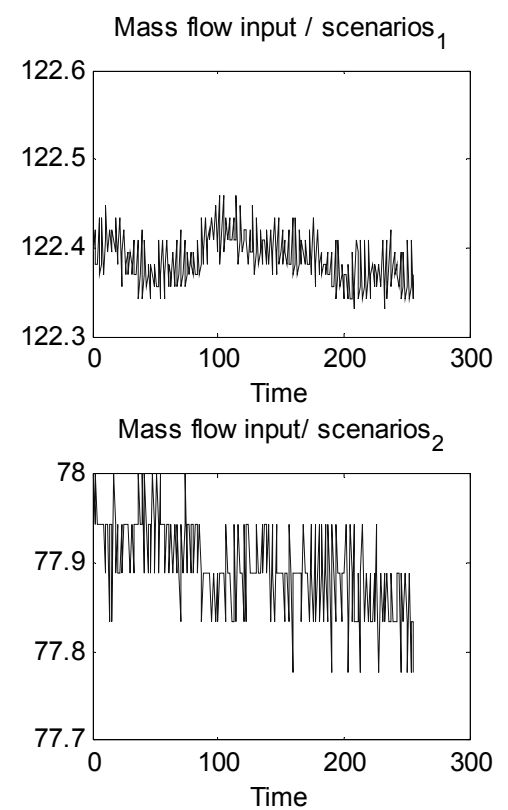

Figure 9 shows the response of the compression system for the pressure input and the associate residual, there is a value that the residuals consistently exceed the threshold of detection, this figure show a case where a surge is detected in the examined compression system.
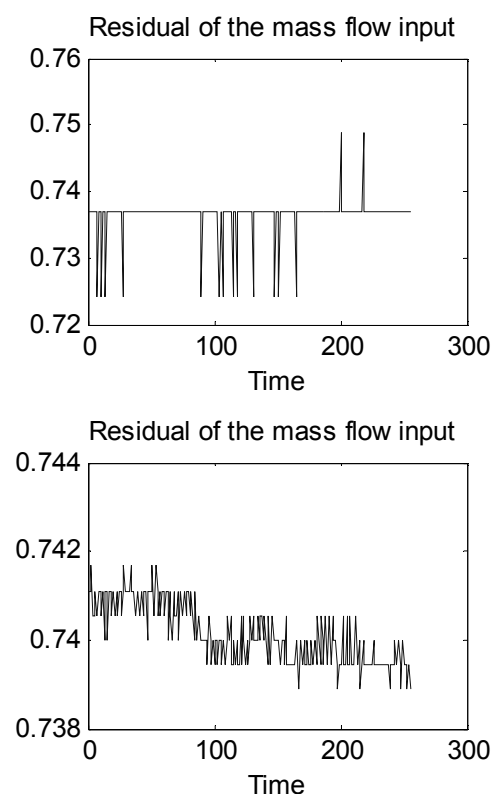

Figure 8. Response of the compression system for the mass flow input with surge mode operation
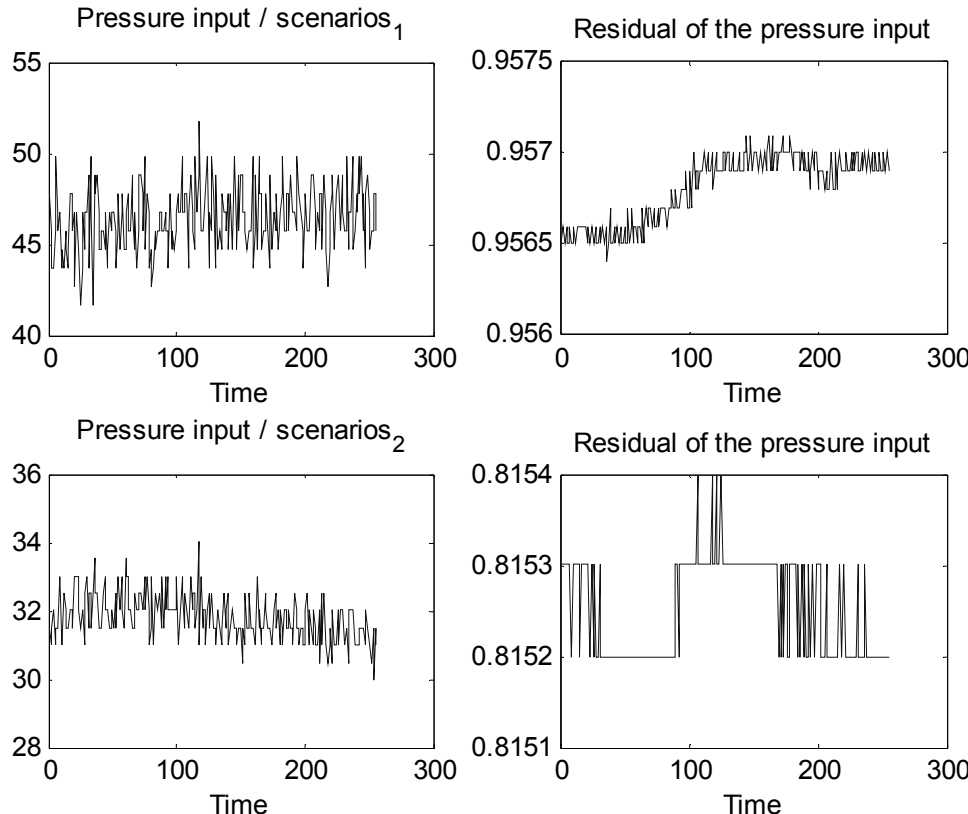

Figure 9. Response of the compression system for the pressure input with surge mode operation 
The response of the compression system with control of surge using fuzzy observer for surge detection and isolation is examined. Figure 10 shows the response of the compression system for the mass flow input and the associate residual, in this case, the behavior of our compression system is considered nominal (with control of surge). The value of the residual signals is exactly zero.
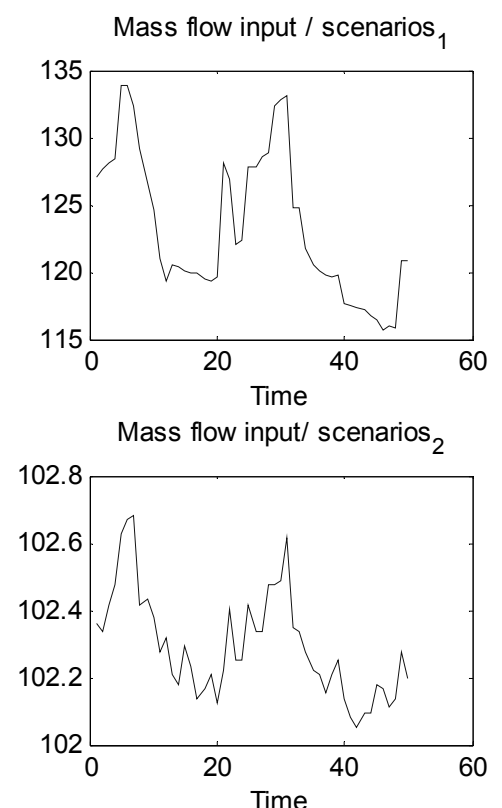

The response of the compression system with control of surge using fuzzy observer for surge detection and isolation is examined. Figure 11 shows the response of the compression system for the pressure input and the associate residual, in this case, the behavior of our compression system is considered nominal (with control of surge).
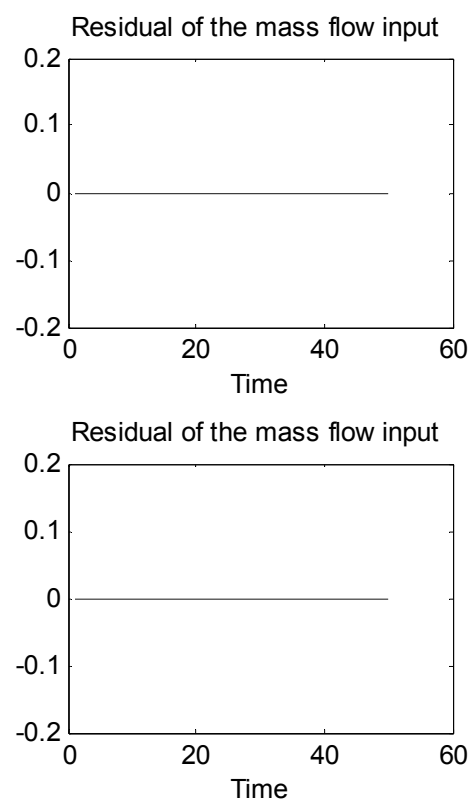

Figure 10. Response of the compression system for the mass flow input with surge control using fuzzy observer
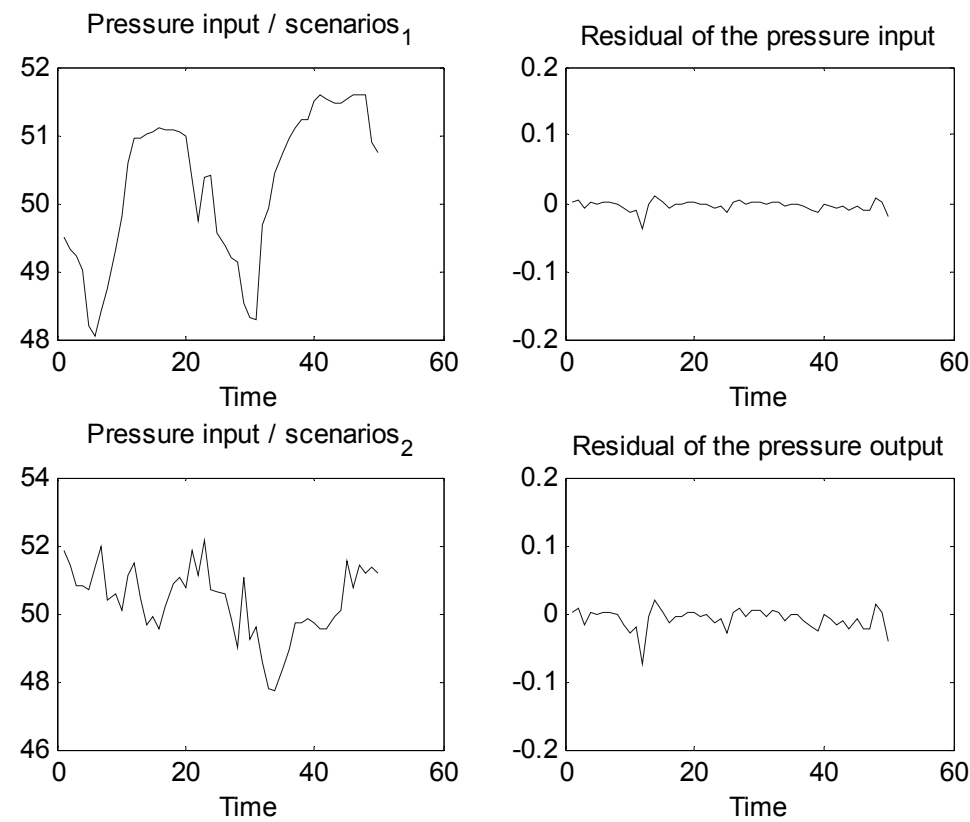

Figure 11. Response of the compression system for the pressure input with surge control using fuzzy observer 
The value of the residual signals is in the threshold of detection, is accepted and indicates that a non detection of surge, due to model error, signal noise, and disturbances, this residual signal is nonzero.

In this section, the proposed methodology is applied to the surge control in compression system. The same steps presented here should be followed in the development of a fuzzy controller system for any dynamic system to which the methodology is suitable.

\section{Conclusion}

In this paper, the new active surge control system is proposed to both protect the centrifugal compression system and reduce the downtime and productivity costs to save the maintenance costs. The paper proposed a new fuzzy controller for surge problem in centrifugal compression system, where human knowledge is used to define the goals and the constraints of the control system. The effectiveness of the proposed methodology is illustrated by the experimental study, performed with the surge in a centrifugal compression system problem. The study showed the effectiveness of such robust design of surge controller based on fuzzy techniques. In this practical application, surge stabilization using the proposed fuzzy controller has been successfully applied and experimentally investigated in the examined compression system (gas compression station in Algeria SC3/Sonatrach), the proposed control technique is very promising and has a good performance.

\section{REFERENCES}

1. ABDEL-HADY, F., S. ABUELENIN, Design and Simulation of a FuzzySupervised PID Controller for a Magnetic Levitation System, Studies in Informatics and Control Journal, ICI Publishing House, vol. 17, no. 3, 2008, pp. 315-328.

2. BENREJEB, M., D. SOUDANI, A. SAKLY, P. BORNE, New Discrete TSK Fuzzy Systems Characterization and Stability Domain. International Journal of Computers, Communications \& Control, Agora University Editing House
- CCC Publications, vol. 1, no. 4, 2006, pp. 9-19.

3. DiEulot, J. Y., Fuzzy Control and Estimation Using Model Inversion. Studies in Informatics and Control Journal, ICI Publishing House, vol. 13, no. 3, 2004, pp. 153-160.

4. DIEULOT, J. Y., P. BORNE, Inverse Fuzzy Sum-product Composition and Its Application to Fuzzy Linguistic Modelling. Studies in Informatics and Control SIC Journal, ICI Publishing House, vol. 14, no. 2, 2005, pp. 73-78.

5. GALINDO, J. R. SERRANO, H. CLIMENT, A. TISEIRA, Experiments and Modelling of Surge in Small Centrifugal Compressor for Automotive Engines, Experimental Thermal and Fluid Science, Elsevier, vol. 32, no. 3, 2008, pp. 818-826.

6. GRAVDAHL, J. T., O. EGELAND, S. O. VATLAND, Drive Torque Actuation in Active Surge Control of Centrifugal Compressors, Automatica, Elsevier, vol. 38, no. 11, 2002, pp. 1881-1893.

7. GRAVDAHL, J. T., F. WILLEMS, B. JAGER DE, O. EGELAND, Modelling of Surge in Free-spool Centrifugal Compressors: Experimental Validation, Journal of Propulsion and Power, Elsevier, vol. 20, no. 5, 2004, pp. 849-857.

8. HAFAIFA, A., K. LAROUSSI, F. LAAOUAD, Robust Fuzzy Fault Detection and Isolation Approach Applied to the Surge in Centrifugal Compressor Modelling and Control, International Journal of Fuzzy Information and Engineering, Springer, vol.2 no.1, 2010, pp.49-73.

9. HAFAIFA, A., F. LAAOUAD, K. LAROUSSI, Fuzzy Approach Applied in Fault Detection and Isolation to the Compression System Control, Studies in Informatics and Control Journal, ICI Publishing House, vol. 19, no. 1, 2010, pp. 17-26.

10. HAFAIFA, A., F. LAAOUAD, K. LAROUSSI, Fuzzy Logic Approach Applied to the Surge Detection and Isolation in Centrifugal Compressor, Automatic Control and Computer 
Sciences Journal, Springer, vol. 44, no. 1, 2010, pp. 53-59.

11. HAFAIFA, A., F. LAAOUAD, M. GUEMANA, A New Engineering Method for Fuzzy Reliability Analysis of Surge Control in Centrifugal Compressor, American Journal of Engineering and Applied Sciences, Science Publisher Inc, vol. 2, no. 4, 2009, pp. 676-682.

12. HAFAIFA, A, F LAAOUAD, K LAROUSSI, Fuzzy Modelling and Control for Detection and Isolation of Surge in Industrial Centrifugal Compressors, Journal of Automatic Control, University of Belgrade, vol.19, no.1, 2009, pp.19-26.

13. HAFAIFA, A., F. LAAOUAD, A. ALEB, Advanced Methods for Timevarying and Nonlinear Processes Using Fuzzy Logic, Proceeding of the 8th International Conference on Computer Modelling and Simulation, 8th ICCMS in Oxford UK, no. 155, 2005, pp. 27-33.

14. HELVOIRT, J. V., B. D. JAGER, Dynamic Model Including Piping Acoustics of a Centrifugal Compression System, Journal of Sound and Vibration, Elsevier, vol. 302, no. 1-2, 2007, pp. 361-378.

15. MOISE, G., Applying Fuzzy Control in the Online Learning Systems. Studies in Informatics and Control Journal, ICI Publishing House, vol. 18, no. 2, 2009, pp. 165-172.

16. NAYFEH, M., E. H. ABED, High-gain Feedback Control of Rotating Stall in Axial Flow Compressors, Automatica, Elsevier, vol. 38, no. 6, 2002, pp. 995-1001.
17. PADUANO, J. D., E. M. GREITZER, A. H. EPSTEIN, Compression System Stability and Active Control, Annual Review of Fluid Mechanics, Elsevier, vol. 33, 2001, pp. 491-517.

18. POP, B., I. DZITAC, Fuzzy Control Rules in Convex Optimization. Studies in Informatics and Control Journal, ICI Publishing House, vol. 15, no. 4, 2007, pp. 363-366.

19. POP, B., I. DZITAC, Mixed Variables Fuzzy Programming Algorithm. Studies in Informatics and Control Journal, Publishing House, vol. 16, no. 2, 2007, pp. 185-190.

20. RUDAS, I. J., L. HORVÁTH, Intelligence for Assistance of Engineering Decisions in Modelling Systems, Studies in Informatics and Control Journal, ICI Publishing House, vol. 15, no. 03, 2006, pp. 297-306.

21. SAKLY, A., B. ZAHRA, M. BENREJEB, Stability Study of Mamdani's Fuzzy Controllers Applied to Linear Plants, Studies in Informatics and Control Journal, ICI Publishing House, vol. 17, no. 4, 2008, pp. 441-452.

22. SPAKOVSKY, Z. S., J. B. GERTZ, O. P. SHARMA, J. D. PADUANO, A. H. EPSTEIN, E. M. GREITZER, Influence of Compressor Deterioration on Engine Dynamic Behaviours and Transient Surge Margin, ASME Journal of Turbomachinery, vol. 122, no. 3, 2000, pp. 477-484. 